to themselves under this automorphism. Moreover, it is possible to construct a group of order $2^{m}$, where $m$ is any given number, which admits an automorphism of order 2 in which the number of inverse correspondences is an arbitrary number which satisfies these two conditions and the order of $K$ is any divisor of $2^{m}$. In particular, the number of inverse correspondences under an automorphism of order 2 of any abelian group of odd order is always equal to the order of the corresponding commutator subgroup. In fact, in an automorphism of order 2 of any group whatsoever of odd order a necessary and sufficient condition that an operator corresponds to its inverse is that it is a commutator under this automorphism, but these commutators do not necessarily constitute a group when the given group of odd order is non-abelian.

The University of IlLinois

\title{
THE FOURTH POSTULATES OF RIESZ AND HAUSDORFF*
}

BY ORRIN FRINK JR.

A question which has remained unanswered for some time is whether every space which satisfies the four postulates of Riesz for points of accumulation and the additional postulate that every derived set is closed, is necessarily a Hausdorff space. A paper on this subject was recently presented to the American Mathematical Society. $\dagger$ I have succeeded in constructing an example, given below, which shows that the answer is in the negative.

A good discussion of the two postulate systems of Riesz and Hausdorff may be found in Fréchet's recent Borel monograph, Les Espaces Abstraits. Although Hausdorff's postulates are in terms of neighborhoods and those of Riesz concern the accumulation points directly, there is almost a complete parallelism between them, provided we add to Riesz's four, as Fréchet does, a fifth postulate that every derived set is closed. The only

* Presented to the Society, December 27, 1929.

† R. G. Putnam, this Bulletin, vol. 35 (1929), p. 442. 
uncertainty is caused by the fact that Hausdorff's fourth postulate is apparently slightly stronger than Riesz's number four. The postulates of Riesz are as follows:

1. Every point of accumulation of a set $E$ is a point of accumulation of every set which includes $E$ as a subset.

2. Every point of accumulation of the sum of two sets is a point of accumulation of at least one of the two sets.

3. A set consisting of a single point has no points of accumulation.

4. Every point of accumulation of a set $E$ is determined by the knowledge of all the subsets of $E$ of which this point is a point of accumulation.

Postulate 4 is interpreted as meaning that if $a$ and $b$ are two distinct points of accumulation of a set $E$, there exists at least one subset of $E$ of which one of the points $a$ and $b$, and one alone, is a point of accumulation.

The fourth postulate of Hausdorff requires that if $x$ and $y$ are any two distinct points, there exist neighborhoods $U_{x}$ and $V_{y}$ of $x$ and $y$ respectively, which have no points in common.

The example is a space consisting of:

I. All points of the $X Y$ plane with positive $x$ and $y$ coordinates. A neighborhood of such a point is defined as usual to consist of the points inside a circle with the point as center and with any radius small enough so that only points with positive $x$ and $y$ coordinates are included.

II. The point $O$ (the origin). A neighborhood of $O$ is defined to be a quarter circle consisting of $O$ and all points with positive $x$ and $y$ coordinates lying inside a circle with $O$ as center.

III. The point $P$. This point is given abstractly and may be thought of as replacing all positive points of the $x$-axis. A neighborhood of $P$ by definition is to consist of $P$ and all points lying beneath some curve $y=f(x)$, where $f(x)$ is a continuous, single valued, everywhere positive function defined for all positive values of $x$. The points $O$ and $P$ are not contained in any neighborhoods but their own.

From this definition it follows that $O$ is an accumulation point of a set when and only when the set contains a sequence of points converging in the ordinary sense to $O$, and $P$ is an accumulation point of a set when and only when the set contains a sequence of points converging in the ordinary sense to some 
positive point of the $X$-axis (which isn't there, of course, in our space). A sequence of points converging to $O$, however, cannot have $P$ for an accumulation point. For we can select our curve $y=f(x)$ in such a way that all points of the sequence other than $P$ lie above it.

This space satisfies all the postulates of Riesz when accumulation points have been defined as usual in terms of neighborhoods. Postulate 1 is satisfied in every neighborhood space. Postulate 2 follows from the fact that given any two neighborhoods of a point there is a third neighborhood contained in them both. Postulate 3 follows from the fact that given any two points there is a neighborhood of one of them that does not contain the other, and conversely. Riesz's postulate 4 is also satisfied. This requires that we be able to distinguish between two accumulation points of a set by finding a subset of which one is an accumulation point and the other not. If neither of these two points is $O$ or $P$, we obviously have this property, since our space is cartesian if we omit $O$ and $P$. In fact, any set of which a given point is an accumulation point has a subset of which this point is the unique accumulation point. But even if one of the points is $O$ or $P$, we have seen above that if either of these two points is an accumulation point of a set $M$, it is the unique accumulation point of a sequence of points picked out of $M$. The fifth postulate, that every derived set is closed, follows from the fact that our neighborhoods are open sets.

However, Hausdorff's fourth postulate is not satisfied. For every neighborhood of $O$ has points in common with every neighborhood of $P$. Furthermore, this will be true if we replace our definition of neighborhoods by any equivalent definition (equivalent in the sense of leading to the same definition of accumulation points). For every new neighborhood would have to contain an old neighborhood as a subset. The essential feature of this example is then that although all neighborhoods of $O$ and $P$ have points in common, we can always distinguish between $O$ and $P$ as accumulation points of a set by finding a subset of which one is an accumulation point but not the other.

Pennsylvania State College 